\title{
Photothermal alternative to device fabrication using atomic precision advanced manufacturing techniques
}

\author{
Aaron M. Katzenmeyer $\odot$, ${ }^{\text {a,* }}$ Sanja Dmitrovic, ${ }^{\mathrm{b}}$ Andrew D. Baczewski $\odot^{\mathrm{a}}{ }^{\mathrm{a}}$ \\ Quinn Campbell, ${ }^{\mathrm{a}}$ Ezra Bussmann $\odot,{ }^{\mathrm{a}}$ Tzu-Ming Lu, ${ }^{\text {a }}$ Evan M. Anderson $\odot,{ }^{\mathrm{a}}$ \\ Scott W. Schmucker $\odot$, Jeffrey A. Ivie, ${ }^{a}$ Deanna M. Campbell, ${ }^{a}$ \\ Daniel R. Ward $\odot,{ }^{a}$ David A. Scrymgeour, ${ }^{\text {a George T. Wang, }}{ }^{a}$ \\ and Shashank Misra ${ }^{\mathrm{a}, *}$ \\ a Sandia National Laboratories, Albuquerque, New Mexico, United States \\ ${ }^{b}$ University of Arizona, College of Optical Sciences, Tucson, Arizona, United States
}

\begin{abstract}
The attachment of dopant precursor molecules to depassivated areas of hydrogenterminated silicon templated with a scanning tunneling microscope (STM) has been used to create electronic devices with subnanometer precision, typically for quantum physics experiments. This process, which we call atomic precision advanced manufacturing (APAM), dopes silicon beyond the solid-solubility limit and produces electrical and optical characteristics that may also be useful for microelectronic and plasmonic applications. However, scanned probe lithography lacks the throughput required to develop more sophisticated applications. Here, we demonstrate and characterize an APAM device workflow where scanned probe lithography of the atomic layer resist has been replaced by photolithography. An ultraviolet laser is shown to locally and controllably heat silicon above the temperature required for hydrogen depassivation on a nanosecond timescale, a process resistant to under- and overexposure. STM images indicate a narrow range of energy density where the surface is both depassivated and undamaged. Modeling that accounts for photothermal heating and the subsequent hydrogen desorption kinetics suggests that the silicon surface temperatures reached in our patterning process exceed those required for hydrogen removal in temperature-programmed desorption experiments. A phosphorus-doped van der Pauw structure made by sequentially photodepassivating a predefined area and then exposing it to phosphine is found to have a similar mobility and higher carrier density compared with devices patterned by STM. Lastly, it is also demonstrated that photodepassivation and precursor exposure steps may be performed concomitantly, a potential route to enabling APAM outside of ultrahigh vacuum. () The Authors. Published by SPIE under a Creative Commons Attribution 4.0 Unported License. Distribution or reproduction of this work in whole or in part requires full attribution of the original publication, including its DOI. [DOI: 10.1117/1.JMM.20.1.014901]
\end{abstract}

Keywords: photolithography; photothermal effects; hydrogen lithography; surface morphology; nanoscale devices; scanned probe lithography.

Paper 20040 received Aug. 9, 2020; accepted for publication Feb. 19, 2021; published online Mar. 6, 2021.

\section{Introduction}

Scanning tunneling microscopy (STM) can be used to incorporate phosphorus dopants at the surface of silicon with \pm 1 lattice site $(\sim 0.35 \mathrm{~nm})$ precision. This capability has been leveraged to fabricate one-off dopant-based physics experiments where proximal nanoscale electrostatic gates are used to manipulate the electrons bound to one or a few well-placed phosphorus atoms. ${ }^{1-5}$ With the exponentially rising development cost of proceeding to smaller device sizes in microelectronics, leveraging this fabrication pathway, which we call atomic precision advanced manufacturing (APAM), to explore digital microelectronics concepts at the physical limit of atoms can provide an important pathfinding function. ${ }^{6-8}$ However, the complexity of devices that can be made with APAM needs to advance significantly and process development will require making an enormous number of devices, a task belabored by STM. In addition, the APAM process

*Address all correspondence to Aaron M. Katzenmeyer, amkatze@sandia.gov; Shashank Misra, smisra@sandia.gov 
introduces a dopant density into silicon that exceeds the solid solubility limit, which creates unique electrical and optical properties ${ }^{6-8}$ that are qualitatively different than what is possible at conventional levels of doping. Here, potential applications, from enhanced doping in microelectronics to plasmonic devices, do not require atomic precision, but need patterning over larger areas than attainable with STM, and a path to manufacturability.

The only step of the APAM process that has no straightforward translation to $300 \mathrm{~mm}$ waferscale processing is hydrogen depassivation lithography. In the APAM process, hydrogen attached to an atomically clean $\mathrm{Si}(100)$ surface is selectively depassivated using the STM with sub-nm precision. ${ }^{9}$ Dopant precursor molecules, such as phosphine, subsequently introduced into the ultrahigh vacuum chamber attach selectively only to the depassivated areas and not to sites that remain passivated with hydrogen. ${ }^{10} \mathrm{~A}$ modest thermal anneal to temperatures that leave the hydrogen resist mask intact is sufficient to partially incorporate phosphorus dopants into the lattice. The dopants are then encapsulated with a silicon overlayer to activate and protect them. The encapsulation step must be executed at low enough temperatures to prevent diffusion of the dopants. ${ }^{11}$ Key parts of the APAM process have been demonstrated below a temperature of $700^{\circ} \mathrm{C}$, low enough that it may leave much of front-end-of-line microelectronics manufacturing intact. Similarly, APAM has been shown to survive processing up to $500^{\circ} \mathrm{C}$, high enough that it may remain intact in the face of much of back-end-of-line microelectronics manufacturing. ${ }^{12}$ To scale the process, the initial sample preparation and hydrogen termination can be implemented at the wafer scale within a low-pressure chemical vapor deposition tool. ${ }^{13}$ Both precursor dosing and low-temperature silicon encapsulation can, in principle, be implemented in a straightforward way at the wafer scale with a molecular beam epitaxy system and a doping antechamber. Thus, the eventual development of a manufacturable APAM workflow appears possible given significant development work. However, even at the scale of a single device on one chip, the time to fabricate a device, typically one day, is dominated by the time it takes STM to depassivate hydrogen.

Fortunately, hydrogen depassivation has previously been demonstrated using several methods that are much faster than scanned probe microscopy. Near-surface secondary electrons from a scanning electron microscope (SEM) have been used to remove hydrogen from a passivated surface with a spot-size of around $200 \mathrm{~nm} .{ }^{14}$ While much faster than scanned probe microscopy, SEM shares the limitation of a serial writing process, as opposed to the parallel writing of many features afforded by photolithography. Mid-infrared light can be used to directly excite the stretching mode of the Si-H bond, but resolution limitations from the wavelength required $(4.8 \mu \mathrm{m})$ limit the applicability of this mechanism. ${ }^{15}$ Direct excitation of the hydrogen from the bonding to the antibonding configuration has been demonstrated with $157 \mathrm{~nm}$ light. ${ }^{16}$ However, optics at vacuum ultraviolet wavelengths are both costly and difficult. At wavelengths between the indirect band gap for silicon $(1100 \mathrm{~nm})$ and the direct electronic excitation $(180 \mathrm{~nm})$, light has been used to locally heat silicon above the temperature where it will desorb from silicon. ${ }^{17}$

Here, we fabricate a simple APAM device using a pulsed ultraviolet laser to photothermally desorb hydrogen. Absorption in silicon is strong for UV photon energies that exceed the direct bandgap. We expect $337 \mathrm{~nm}$ light to localize heat to within $10 \mathrm{~nm}$ of the surface during the laser pulse, and pulsing to limit the total heat deposited in the sample. More common lithography wavelengths - $365,248,193$, and $13.5 \mathrm{~nm}$-could be used by accounting for the wavelength-dependent penetration depth and in the last case additional patterning mechanisms. We first show that the atomic nature of the hydrogen resist ${ }^{18}$ makes it resistant to dose-related issues that lead to under- and overexposure with conventional photoresists. Next, we use STM imaging to evaluate the suitability of the depassivated regions for the phosphine chemistry required to create a device. We find a narrow range of laser energy density where hydrogen is depassivated without disrupting the structure of the surface. ${ }^{19-21}$ A model of the photothermal heating process suggests that the surface temperatures achieved in our experiments are highly nonuniform and difficult to precisely constrain given the nonlinear nature of thermal transport over the range of relevant temperatures. Nevertheless, consideration of the desorption kinetics and the fact that the silicon surface only remains hot for a few ns per pulse suggests that the temperatures that we are achieving must be significantly higher than the $500^{\circ} \mathrm{C}$ peak observed in temperature-programmed desorption (TPD) experiments where dwell time is orders of magnitude longer. ${ }^{22}$ Finally, we present magnetotransport data from van der Pauw structures fabricated using photothermal desorption. 


\section{Methods}

\subsection{Experimental Procedure}

Samples were cleaned and hydrogen terminated by first flashing to $950^{\circ} \mathrm{C}$ in ultrahigh vacuum $\left(1 \times 10^{-10}\right.$ torr) to remove the surface oxide, followed by passivation using $2 \times 10^{-6}$ torr of atomic hydrogen created by a hot tungsten filament. The samples used in Figs. 1-3 were first prepared with this procedure, then exposed to pulsed laser light. In Figs. 1 and 2, the chip was removed to air to form native oxide selectively on depassivated regions, then reinserted into vacuum. An anneal to $300^{\circ} \mathrm{C}$ removed water physisorbed on the surface, and a subsequent $30 \mathrm{~nm}$ silicon cap deposited at the same temperature using a sublimation source was used to preserve the interface. A Nikon optical microscope identifies the contrast created by the buried dielectric in differential interference contrast (DIC) imaging. In Fig. 3, STM images were taken after hydrogen depassivation using an Omicron Variable Temperature STM operating at room temperature. Images shown are all recorded at a sample bias of $-2.5 \mathrm{~V}$ (corresponding to electrons tunneling from the sample to the tip) and with an imaging setpoint of $0.2 \mathrm{nA}$. The sample used in Fig. 5 was fabricated following the procedure found in Ref. 12 with a photothermal process replacing STMbased hydrogen depassivation. The samples were contacted by etching vias into the implants and depositing aluminum metal. Magnetotransport measurements were taken at $4 \mathrm{~K}$ in a maximum field of $1 \mathrm{~T}$. Laser depassivation was performed using a custom-made scanning laser microscope built to interface to UHV chambers described above via a viewport. The laser is mounted on $x, y$, and $z$ motorized stages with long $(50 \mathrm{~mm}$ ) travel range and submicron (less than $30 \mathrm{~nm}$ ) specified resolution. Optics are mounted to the laser such that three-dimensional scanning is possible without movement of the sample. The beampath from the laser includes attenuators, an $x y$ alignment mount, two beamsplitters, and the objective lens. The first beamsplitter is used to provide sample illumination via an LED. The second is used in conjunction with a magnifying lens and camera to enable positioning with respect to sample features. A nitrogen $\left(\mathrm{N}_{2}\right)$ laser emitting $3.5 \mathrm{~ns}$ (FWHM) pulses of $126 \mu \mathrm{J}$ at $337.1 \mathrm{~nm}$ (bandwidth $0.1 \mathrm{~nm}$ ) was used with variable focus and attenuation as the illumination source. The laser pulsing was triggered with a function generator to emit from a single pulse to a repetition rate of $100 \mathrm{~Hz}$ depending on desired exposure. The beam size exiting the laser aperture was $3 \mathrm{~mm} \times 7 \mathrm{~mm}$ with a specified full angle divergence of $5 \mathrm{~mm} \times 8 \mathrm{mrad}$. For this study, a 50-mm focal length planoconvex lens (Figs. 1 and 2) or a 13.3-mm effective focal length reflecting objective was used to focus the light onto the sample. The beam profile for the reflecting objective was measured, subsequent to traversing the viewport, using the on-board camera and is shown in Fig. 4(a). The measurement indicates that the short-axis divergence of the laser is $1 / 3$ of that specified. The long-axis divergence is measured to be as specified. Profiling the focused beam using a scanning pinhole confirmed this observation.

\subsection{Photothermal Heating Model}

To better understand the conditions required for hydrogen depassivation, we use a physical model of photothermal heating that allows us to convert the measured beam profile into a time-dependent surface temperature. A similar model has been previously used in the context of computing the melting threshold of crystalline and amorphous silicon irradiated by a pulsed excimer UV laser. ${ }^{23}$ We consider a planar sample for which the plane of incidence is $z=0$ and silicon occupies the half-space $z \geq 0$. The temperature, $T(x, y, z, t)$, is governed by a heat equation of the form

$$
\left[\rho C \partial_{t}-\nabla(\kappa(T) \nabla)\right] T(x, y, z, t)=\alpha(T)(1-R(T)) I(x, y) \exp (-\alpha(T) z) f(t),
$$

where $\rho$ is the mass density, $C$ is the heat capacity, $\kappa(T)$ is the thermal conductivity, $\alpha(T)$ is the absorption coefficient, and $R(T)$ is the reflectance. A homogeneous Neumann boundary condition (i.e., $\left.\partial_{z} T\right|_{z=0}=0$ ) is applied at the interface between the silicon and vacuum to enforce zero thermal flux between the two domains. The forcing functions, $I(x, y)$ and $f(t)$, respectively correspond to the profile of the incident beam in the plane of the sample and the time-dependence of the pulse. The former is experimentally measured and the latter is taken to be a Gaussian pulse with a 3.5-ns FWHM. 
Equation (1) is numerically solved by making use of a Green's function approach which reduces the time-dependent surface temperature to an integral that is evaluated by quadrature. We note that the temperature dependence of $\alpha$ and $R$ are negligible for the conditions in our experiment, ${ }^{24}$ whereas the temperature dependence of $\kappa$ is not. ${ }^{25}$ Rather than developing a solution for the full nonlinear problem that accounts for the complex spatial heterogeneity of the beam, we simply solve a linearized version of our model assuming a range of fixed values for $\kappa$ and compute the time-dependent surface temperature for exemplary positions within the measured beam profile. Specifically, we consider the thermal conductivity at room temperature and $1500^{\circ} \mathrm{C}$, as well as the geometric mean of those two values. The former are respectively representative of lower and upper bounds on the temperature, whereas the latter is intended to approximate the solution to the nonlinear model. Naturally, this gives us a wide range of temperatures. We note that reality is likely closest to the geometric mean of the two, which is also indicated in our results. Given the precision that we are attempting to achieve in our predictions, the spread of temperatures that we report is acceptable. Specifically, we accept that our model ignores important physics (e.g., melting and nonlinearity in the thermal conductivity), and the results are intended to illustrate the spatial heterogeneity of the pulse and the fact that the predicted temperature is likely higher than the $\mathrm{H}_{2}$ TPD peak at $500^{\circ} \mathrm{C}^{22}$ The latter fact will prove to be important in understanding the observed $\mathrm{H}_{2}$ desorption kinetics.

\section{Results and Discussion}

\subsection{Atomic Resist}

In contrast to photolithography with conventional resists, the concept of dose loses relevance for photothermal patterning of atomic resists chemically bonded to the surface. For photothermal depassivation, the peak temperature of the silicon surface must exceed the temperature at which hydrogen desorbs from silicon. Below this threshold, no hydrogen is desorbed, and above this threshold, there is no penalty for multiple or extended exposures. This "digital" behavior may offer distinct advantages over dose-dependent conventional resists. The time-dependence of the surface temperature for a variety of conditions is shown in Fig. 4(b). It is evident that the surface temperature is only appreciably different from room temperature for a few nanoseconds, corresponding to the pulse duration. In order for hydrogen desorption to occur in such a short time, the temperature has to be sufficiently high that the rate of recombinative desorption into $\mathrm{H}_{2}$ is faster than this time scale. For a pulse that achieves a sufficiently high surface temperature, most of the hydrogen will desorb in a single shot, though we must be careful as to not heat the surface too much to avoid damage, such as amorphization, melting, or ablation. However, as the surface temperature achieved in a single shot moves below this desorption threshold, hydrogen removal will become increasingly rare. The cooling rate of the sample is sufficiently fast that the "dose" cannot accumulate as it would in a conventional photoresist, and very little hydrogen is desorbed.

In practice, there is no easy way to measure the temperature of just the surface of silicon for the several nanoseconds ( 3.5 ns FWHM) it is being irradiated, so instead, we track the energy density of the laser and use a photothermal heating model to arrive at a putative range of temperatures. Both the concept of subthreshold exposure desorbing no hydrogen and multiple abovethreshold exposures causing no harm are illustrated in the data of Figs. 1 and 2.

Here, we use differential interference optical microscopy to show the dielectric contrast formed by native oxide formation in depassivated areas of the surface, as described in Sec. 2. This is a quick way of narrowing down the large phase space of illumination parameters to favorable ranges that show depassivation. In Fig. 1, the black box indicates a below threshold (below $\mathrm{T}_{\text {desorb }}$ ) exposure where no patterning has occurred. A slight increase in laser energy, by $10 \%$, results in hydrogen desorption and native oxide formation (red box). Unfortunately, systematic errors in the determination of the area of the focused pulse complicate extraction of a threshold energy density for hydrogen depassivation from this data. Figure 2 shows the effect of multiple above-threshold exposures. Despite a comparable energy density in Figs. 2(a) and 2(b), there is no damage being done to the silicon from multiple exposures. Moreover, multiple exposures are seen to reduce pattern roughness that likely results from statistical fluctuations of the light source. 


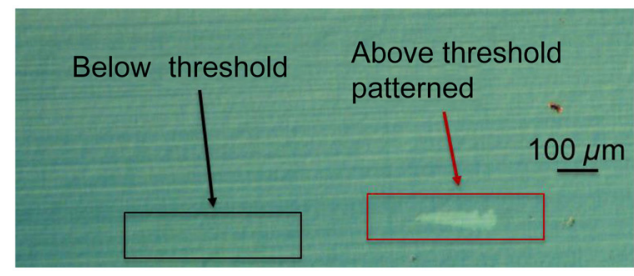

Fig. 1 DIC microscopy image of an exposure test sample. The red box contains an oxidized region resulting from above threshold exposure. The black box contains a region exposed with insufficient energy density to reach the desorption temperature. Hence, no oxidation occurred and no contrast is observed with respect to the surrounding area.

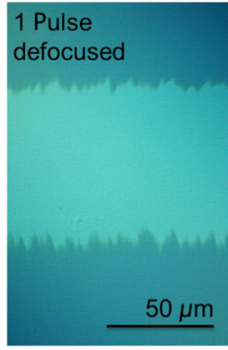

(a)

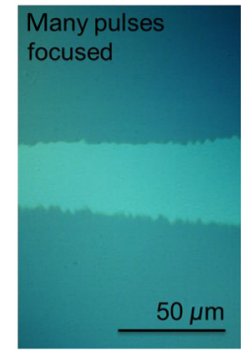

(b)

Fig. 2 100x DIC microscopy image of an oxidized sample patterned by (a) a single laser pulse and (b) many laser pulses. Patterning with many pulses can potentially reduce edge roughness without overexposure.

\subsection{Surface Roughening}

There is a potentially wide window of surface temperatures where photothermal hydrogen desorption can be expected to be effective. FTIR experiments suggest that the temperature must be at least $430^{\circ} \mathrm{C}$ to activate desorption of the monohydride termination, ${ }^{17}$ but below $1400^{\circ} \mathrm{C}$ to avoid melting. ${ }^{26}$ Due to the kinetics of recombinative desorption and the rapid cooling of the surface subsequent to the laser pulse, we find that we need to get closer to the melting temperature than the $430^{\circ} \mathrm{C}$ threshold would suggest. However, for the purposes of making a device using the APAM process flow, this surface must also be suitable for phosphine chemistry. The requirement that the atomic-scale $2 \times 1$ dimer-row surface reconstruction of $\mathrm{Si}(100)$ is present may place a significantly tighter constraint on the range of acceptable laser energy density, especially as nonthermal roughening mechanisms may be relevant during a laser pulse.

As shown in Fig. 3, following exposure with fluences of (a) $269 \mathrm{~mJ} \mathrm{~cm}^{-2}$ and (b) $241 \mathrm{~mJ} \mathrm{~cm}^{-2}$, the surface roughens considerably on the atomic scale, which may be advantageous for ultradoping. While the crystallinity of the surface is preserved, as indicated by the layer-by-layer character of the roughening and the $2 \times 1$ dimer rows apparent in the images, such roughening is generally undesirable for atomic-precision device fabrication. As the laser fluence is decreased to (c) $194 \mathrm{~mJ} \mathrm{~cm}^{-2}$, the surface is depassivated while the underlying flat step-terrace surface structure, and the $2 \times 1$ dimer rows remain intact as in STM patterning. As we will show below with a model that treats photothermal heating, these energy densities plausibly raise the surface temperature to the equilibrium melting point of silicon or beyond in certain regions of the beam. However, the rapid cooling of the surface might also mean that this transformation is incomplete, potentially explaining the roughening seen in Figs. 3(a) and 3(b).

\subsection{Surface Temperature Estimates and Desorption Kinetics}

In Fig. 4, we give predictions for the range of time-dependent surface temperatures that are realized in our experiments. These results are based on the numerical solution to a linearization of Eq. (1), in which the temperature-dependence of the thermal conductivity is the only source of 


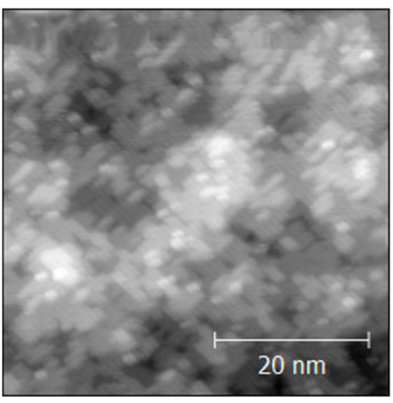

(a)

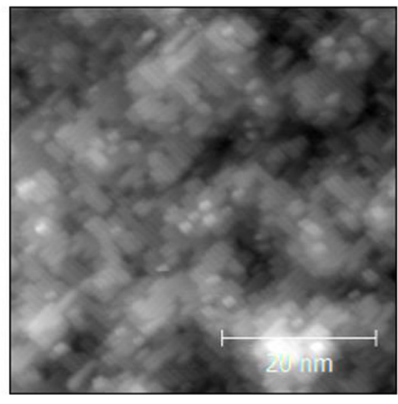

(b)
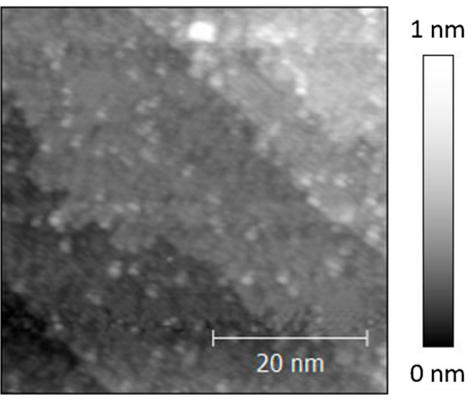

(c)

Fig. $350 \mathrm{~nm} \times 50 \mathrm{~nm}$ STM topography scans of regions exposed with energy densities of (a) $269 \mathrm{~mJ} \mathrm{~cm}^{-2}$, (b) $241 \mathrm{~mJ} \mathrm{~cm}^{-2}$, and (c) $194 \mathrm{~mJ} \mathrm{~cm}^{-2}$. STM lithography in a serpentine pattern was attempted prior to each scan and indicates $\mathrm{H}$ was already removed from the surface by laser exposure.

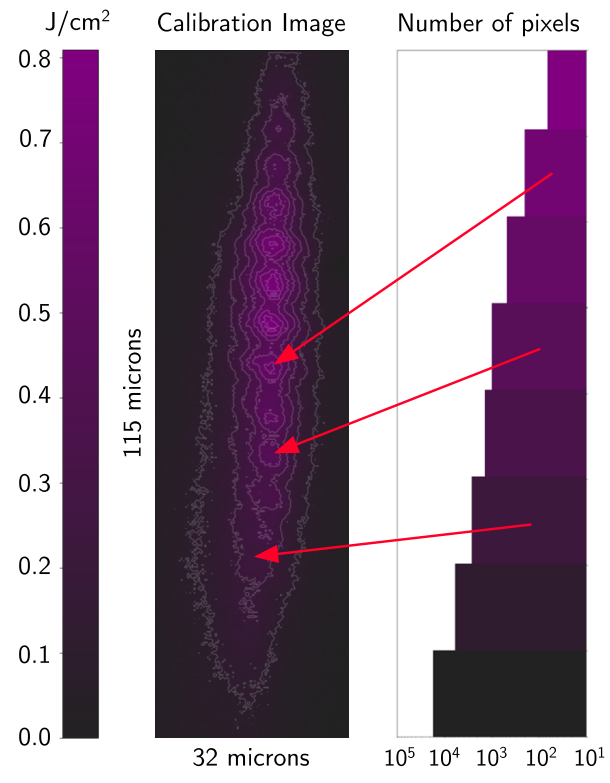

(a)
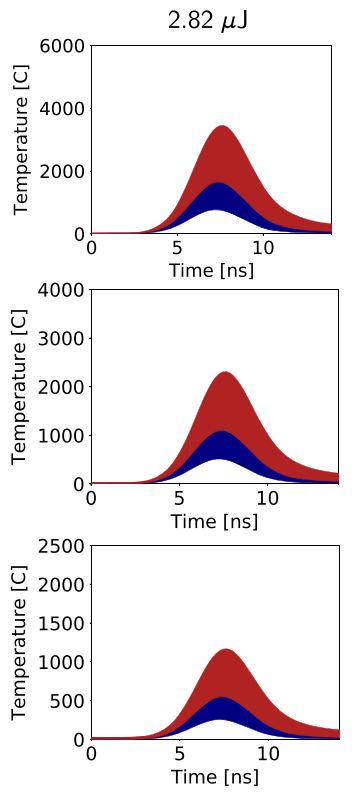

(b)
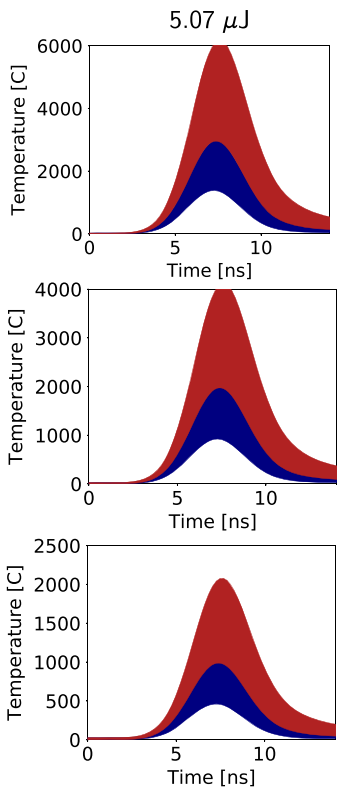

(c)

Fig. 4 Surface temperatures for different positions within the beam for conditions corresponding to two exemplary pulse energies. (a) The measured areal energy density of the calibration pulse with a total energy of $6.5 \mu \mathrm{J}$ and a histogram illustrating the number of pixels corresponding to the indicated range of areal energy densities. (b) Time-dependent surface temperatures based on a rescaling of the calibration image for a total energy of $2.82 \mu \mathrm{J}$, coinciding with beam profile measurements. (c) Time-dependent surface temperatures based on a rescaling of the calibration image for a total energy of $5.07 \mu \mathrm{J}$, coinciding with beam profile measurements. In (b) and (c), we illustrate the surface temperature at pixels typical of three different intensities occurring within the calibration pulse. The corresponding calibration pixels are indicated with red arrows associated with relative frequency in the histogram in (a). Our predictions are based on a numerical solution of a linearized version of Eq. (1) for the room temperature $\left(1.4 \mathrm{~W} \mathrm{~cm}^{-1} \mathrm{~K}^{-1}\right)$ and $1500^{\circ} \mathrm{C}\left(0.2 \mathrm{~W} \mathrm{~cm}^{-1} \mathrm{~K}^{-1}\right)$ thermal conductivities, indicative of the range of relevant values and serving as lower and upper bounds on the surface temperature. The red and blue regions correspond to temperatures above and below the geometric mean of the two thermal conductivities. For instances that do not exceed the melting temperature, we expect the actual temperature to be close to this boundary.

nonlinearity. Rather than treating the nonlinearity explicitly, we simply present the solution to a linear model for the entire range of thermal conductivities that are relevant to crystalline silicon. We expect that a solution to the full nonlinear model would give temperatures close to the geometric mean of the two extremes, which is indicated by the boundary between the red and blue 
regions. The heterogeneity of the surface temperature is accounted for by considering a spatially resolved measurement of the areal energy density which had a total energy of $6.5 \mu \mathrm{J}$. We then present results for three exemplary intensities realized within the beam that are based on rescaling the calibration data for total energies of 2.82 and $5.07 \mu \mathrm{J}$, both of which were realized in experiments in which photothermal depassivation occurred. A histogram for the calibration intensity is provided to give a sense for what fraction of the beam corresponds to each of these intensities.

The surface temperatures illustrated are loose lower and upper bounds with reality likely lying close to the boundary between the red and blue regions. The key takeaways for these loose bounds are that (1) the temperature is only appreciably above room temperature for $\sim 5 \mathrm{~ns}$ and (2) over large fractions of the illuminated area in both experiments the predicted temperatures exceed the $500^{\circ} \mathrm{C}$ TPD peak associated with $\mathrm{H}_{2}$ desorption. Both points are important because desorption kinetics dictate that the temperature must be appreciably above $500^{\circ} \mathrm{C}$ for desorption to occur within $\sim 5 \mathrm{~ns}$, relative to the much longer time scales associated with a TPD measurement. In fact, based on an Arrhenius model using the recombinative desorption barrier from Ref. 22 and a range of plausible attempt frequencies, our observed desorption can only be rationalized if the peak surface temperature is at or above $1100^{\circ} \mathrm{C}$. Other kinetic effects related to the observed surface roughening will require more detailed experiments and modeling to understand.

Relative to our original proceedings article in which some of these results were first reported, ${ }^{27}$ our model and interpretation have changed in light of a more precise quantitative knowledge of the beam profile and total energy per pulse. Previously, we indicated that the condition for desorption appeared to be simply exceeding the temperature associated with the TPD peak. More careful consideration of the desorption kinetics suggests that we must exceed it by nearly a factor of 2 to rationalize the rapid hydrogen removal that is occurring.

\subsection{APAM-Inspired Device}

Having established a window within which photothermal depassivation produces a surface suitable to incorporate phosphine, we now turn to fabrication of a simple van der Pauw device to determine the density of active carriers and their mobility. Many of the interesting electrical and optical properties ${ }^{6}$ of APAM systems derive not from the atomic-scale dimensions, but from the active dopant density being an order of magnitude higher than can be achieved with other techniques. This level of doping - nearly one in every four atoms at the surface-far exceeds the solid solubility limit. To do this, we have photothermally depassivated the hydrogen in the central region of a sample with eight preimplanted contacts in three overlapping exposures, each of energy density $270 \mathrm{~mJ} \mathrm{~cm}^{-2}$ [Fig. 5(a)]. The depassivated area was not constrained to be just the central, square area, but is an inchoate region that extends out to the black alignment marks. While unconventional, this permits a measurement of the carrier density and mobility using the

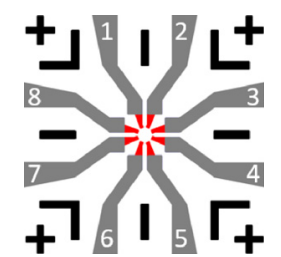

(a)

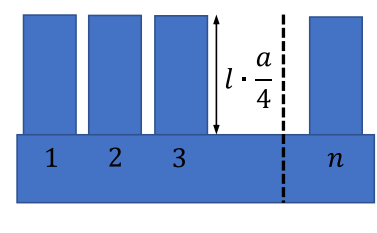

(b)

Fig. 5 (a) Schematic diagram, overhead view, of the van der Pauw device. Black objects are tungsten alignment marks, red objects are ion-implanted contacts, and gray objects are the aluminum contacts that neck in to the implants. The depassivated region, and hence the phosphorus device, was an irregular region larger than the implants but smaller than the alignment marks. Contacts 1, 3, 5, and 7 were used for the measurement, and the other contacts were left open circuit. (b) Cross-sectional view of the scheme considered for calculation of surface area. The height of the pillars is the product of the number of atomic step layers, I, and the atomic step height - one quarter the Si lattice constant, $a$, as measured by STM. 
van der Pauw method. The phosphorus delta layer was electrically characterized at $4 \mathrm{~K}$, at which the carriers in the substrate freeze out and do not contribute to transport. DC and low-frequency transport measurements were performed in a magnetic field for extracting the density and mobility of the delta layer. The carrier density extracted from the Hall coefficient was $4.8 \times 10^{14} \mathrm{~cm}^{-2}$. A sheet resistivity of $250 \Omega / \square$ was obtained using the van der Pauw method. From the carrier density and resistivity, we extracted a mobility value of $52 \mathrm{~cm}^{2} \mathrm{~V}^{-1} \mathrm{~s}^{-1}$. This mobility value is consistent with typical phosphorus delta layers produced by the conventional APAM method, while the carrier density is 2.4 times that of a saturated delta layer. ${ }^{8}$ The unconventional geometry of the doped region with respect to the ion-implanted contacts can complicate a van der Pauw measurement. But a potential contributor to the higher carrier density is atomic-scale roughening, as observed in Figs. 3(a) and 3(b), which provides greater surface area for phosphine adsorption. While a simplification compared to the measured topography, we consider a pillar scheme shown in cross-section in [Fig. 5(b)] for calculation. Relevant parameters may be incorporated while avoiding complications arising from curved feature parameterization. An $n \times n$ array with $80 \%$ fill per side yields a surface area 2.2 times that of an atomically flat (10 $\mu \mathrm{m} \times$ $10 \mu \mathrm{m}$ ) device area for $n=4000$ and seven atomic step layers (i.e., the number of steps observed above). While these data are conclusive evidence that APAM works with photolithography replacing STM lithography, additional work is needed to fully understand the origin of the high carrier density. Future studies are also needed to probe the lateral resolution limit obtainable with photopatterning and in particular photothermal patterning as the present demonstration's conditions precluded a spot-size on the order of the wavelength used. In producing dopant features, it is important to consider that two aspects contribute to the critical lateral dimension: (1) a spatiotemporal thermal profile in Si will be determined by the incident light's intensity profile, penetration depth, and pulse duration. The surface temperature profile is proportional to that of the incident beam intensity and that profile rapidly decays in space and time. Depassivation kinetics of the $\mathrm{H}$ resist are dictated by exponential dependencies on temperature and duration. (2) Doping by $\mathrm{PH}_{3}$ requires six adjacent $(3 \times 2$ arrangement) depassivation sites. We estimate for a diffraction-limited Gaussian beam at our wavelength, a 3.5-ns pulse duration, and 10 pulses delivered at the $10 \%$ depassivation level per pulse, that the full width at $50 \%$ depassivation of the final pattern is $30 \mathrm{~nm}$. This level is already probabilistically unlikely to yield a $3 \times 2$ arrangement of open sites. Therefore, deeply subwavelength features seem plausible. Though rivaling the resolution of STM, if possible, would be a far from trivial challenge.

\subsection{Dope-as-You-Go Device}

We have also fabricated van der Pauw structures via a so-called "dope-as-you-go" approach. This method uses an ambient of the flowing dopant precursor gas during the photodepassivation of the Si monohydride. While this shortens the fabrication process, combining two steps into one, a perhaps more important aspect is that successful device fabrication suggests the possibility of moving out of ultrahigh vacuum for APAM processes. The van der Pauw measurements of such a fabricated device at $4 \mathrm{~K}$ show that the process indeed works. The device has a measured mobility of $52 \mathrm{~cm}^{2} \mathrm{~V}^{-1} \mathrm{~s}^{-1}$ and an extremely high carrier density of $6 \times 10^{14} \mathrm{~cm}^{-2}$. It is expected that the extreme carrier density is in part due to effects seen in the standard photolithographic device. It is also possible that laser heating is responsible for additional dopant incorporation.

\section{Conclusion}

In this work, we have shown the viability of photothermal hydrogen lithography to selectively dope parts of the silicon surface using the phosphine chemistry that underlies APAM. For this to open the door both to the rapid fabrication pathway required for process development and applications which leverage dopant densities which exceed the solid solubility limit, two issues remain to be resolved. First, the threshold laser energy density for depassivation needs to be determined, as this is most likely to produce the least amount of surface damage. Fabrication of van der Pauw structures where the laser energy density is systematically stepped above this 
threshold should help determine the role of surface roughness or intrinsic differences in photoand STM lithographic devices. These data and further development of models which also consider disorder nucleation and melting are needed to refine our understanding of the underlying physics. Second, the spot size of the laser needs to be improved either by bringing fiber-coupled laser light close to the sample surface or, more likely, utilizing in-chamber optics. The demonstration of photothermal hydrogen patterning in a direct-write mode with energy densities between 200 and $300 \mathrm{~mJ} \mathrm{~cm}^{-2}$ indicates that photomask-based desorption could be performed rapidly on $300 \mathrm{~mm}$ wafers using commercially available excimer sources. Lastly, while this work focuses on hydrogen desorption from $\mathrm{Si}(100)$ and phosphine doping, the general technique of photothermal modification of the near-surface region could be widely useful for other substrates, resists, and dopant precursors.

\section{Acknowledgments}

This work was supported by the Laboratory Directed Research and Development Program at Sandia National Laboratories and was performed, in part, at the Center for Integrated Nanotechnologies, a U.S. DOE, Office of Basic Energy Sciences user facility. Sandia National Laboratories is managed and operated by National Technology and Engineering Solutions of Sandia, LLC, a wholly owned subsidiary of Honeywell International, Inc., for the U.S. Department of Energy under contract DE-NA-0003525. The views expressed in the article do not necessarily represent the views of the U.S. DOE or the United States Government.

\section{References}

1. M. Fuechsle et al., "A single-atom transistor," Nat. Nanotechnol. 7(4), 242-246 (2012).

2. D. Keith et al., "Single-shot spin readout in semiconductors near the shot-noise sensitivity limit," Phys. Rev. X 9(4), 041003 (2019).

3. Y. He et al., "A two-qubit gate between phosphorus donor electrons in silicon," Nature 571(7765), 371-375 (2019).

4. M. Koch et al., "Spin read-out in atomic qubits in an all-epitaxial three-dimensional transistor," Nat. Nanotechnol. 14(2), 137-140 (2019).

5. J. Wyrick et al., "Atom-by-atom fabrication of single and few dopant quantum devices," Adv. Funct. Mater. 29(52), 1903475 (2019).

6. D. R. Ward et al., "Atomic precision advanced manufacturing for digital electronics," Electron. Dev. Failure Anal. 22(1), 4-11 (2020).

7. E. M. Anderson et al., "Low thermal budget high-k/metal surface gate for buried donorbased devices," J. Phys. Mater. 3(3), 035002 (2020).

8. A. M. Katzenmeyer et al., "Assessing atomically thin delta-doping of silicon using midinfrared ellipsometry," J. Mater. Res. 35(16), 2098-2105 (2020).

9. T.-C. Shen et al., "Atomic-scale desorption through electronic and vibrational excitation mechanisms," Science 268(5217), 1590-1592 (1995).

10. S. Schofield et al., "Atomically precise placement of single dopants in Si," Phys. Rev. Lett. 91(13), 136104 (2003).

11. L. Oberbeck et al., "Measurement of phosphorus segregation in silicon at the atomic scale using scanning tunneling microscopy," Appl. Phys. Lett. 85(8), 1359-1361 (2004).

12. D. R. Ward et al., "All-optical lithography process for contacting nanometer precision donor devices," Appl. Phys. Lett. 111(19), 193101 (2017).

13. T. Škereň et al., "CMOS platform for atomic-scale device fabrication," Nanotechnology 29(43), 435302 (2018).

14. T. Hallam et al., "Use of a scanning electron microscope to pattern large areas of a hydrogen resist for electrical contacts," J. Appl. Phys. 102(3), 034308 (2007).

15. Z. Liu et al., "Desorption of $\mathrm{H}$ from $\mathrm{Si}(111)$ by resonant excitation of the $\mathrm{Si}-\mathrm{H}$ vibrational stretch mode," Science 312(5776), 1024-1026 (2006).

16. A. Pusel, U. Wetterauer, and P. Hess, "Photochemical hydrogen desorption from H-terminated silicon (111) by VUV photons," Phys. Rev. Lett. 81(3), 645 (1998). 
17. P. Gupta, V. Colvin, and S. George, "Hydrogen desorption kinetics from monohydride and dihydride species on silicon surfaces," Phys. Rev. B 37(14), 8234 (1988).

18. J. Randall et al., "Atomic precision patterning on $\mathrm{Si}$ : an opportunity for a digitized process," Microelectron. Eng. 87(5-8), 955-958 (2010).

19. J. Xu, S. Overbury, and J. Wendelken, "Selective laser removal of the dimer layer from Si(100) surfaces revealed by scanning tunneling microscopy," Phys. Rev. B 53(8), R4245 (1996).

20. J. Kanasaki et al., "Primary processes of laser-induced selective dimer-layer removal on si(001)-(2×1)," Phys. Rev. Lett. 89(25), 257601 (2002).

21. K. Yasui and J. Kanasaki, "Scanning tunneling microscopic studies of laser-induced modifications of Si (001)-(2×1) surface," J. Appl. Phys. 110(10), 103516 (2011).

22. H. Wilson et al., "Thermal dissociation and desorption of $\mathrm{PH} 3$ on $\mathrm{Si}(001)$ : a reinterpretation of spectroscopic data," Phys. Rev. B 74(19), 195310 (2006).

23. C. Ong, E. Sin, and H. Tan, "Heat-flow calculation of pulsed excimer ultraviolet laser's melting of amorphous and crystalline silicon surfaces," J. Opt. Soc. Am. B 3(5), 812-814 (1986).

24. G. Jellison, Jr. and F. Modine, "Optical functions of silicon between 1.7 and $4.7 \mathrm{ev}$ at elevated temperatures," Phys. Rev. B 27(12), 7466 (1983).

25. A. Bell, "Review and analysis of laser annealing," RCA Rev. 40, 295-338 (1979).

26. D. Korfiatis, K. T. Thoma, and J. Vardaxoglou, "Conditions for femtosecond laser melting of silicon," J. Phys. D Appl. Phys. 40(21), 6803 (2007).

27. A. Katzenmeyer et al., "Photothermal alternative to device fabrication using atomic precision advanced manufacturing techniques," Proc. SPIE 11324, 113240Z (2020).

Biographies of the authors are not available. 